NOUVELle

\section{Développement des neurones à GnRH dans le cerveau d'embryons humains}

\author{
Anne-Laure Barbotin ${ }^{1,2}$, Vincent Prévot ${ }^{1,3}$, Paolo Giacobini, ${ }^{1,3}$
}

> Chez les mammifères, la fonction de reproduction est sous le contrôle d'une petite population de neurones située dans une région spécifique du cerveau: I'hypothalamus. Ces neurones se projettent au niveau de l'éminence médiane, où ils déversent dans la circulation porte hypophysaire la gonadolibérine ou $\mathrm{GnRH}$ (hormone de libération des gonadotrophines hypophysaires). Cette dernière sera ensuite transportée vers l'antéhypophyse afin de commander la sécrétion des gonadotrophines, l'hormone lutéinisante (LH) et l'hormone folliculo-stimulante (FSH), qui vont réguler à leur tour l'activité gonadique en agissant sur leurs organes cibles, les testicules et ovaires $[1,2](\rightarrow)$.

$\rightarrow$ Voir la Nouvelle de S. Catteau-Jonard et al., $m / s n^{\circ} 5$, mai 2016, page 441

L'ontogenèse des neurones à $\mathrm{GnRH}$ et du système olfactif sont intimement liées. Ce lien étroit est retrouvé en pathologie humaine dans le syndrome de Kallmann. Cette dysplasie olfactogénitale, liée à un défaut de développement du système olfactif et de la migration des neurones à $\mathrm{GnRH}$, associe en effet un hypogonadisme hypogonadotrope et une anosmie. Alors que le développement des neurones à $\mathrm{GnRH}$ et les différentes étapes de leur migration ont été largement documentés dans l'espèce animale (chez le rongeur), les données publiées restent rares chez l'homme, principalement en raison de l'accessibilité restreinte aux tissus embryonnaires humains. Ces données humaines portent, de plus, sur un nombre restreint d'embryons à un stade très précoce du développement.
De nombreuses interrogations subsistent encore concernant le développement du système à GnRH chez l'homme telles que: Où naissent les cellules à $\mathrm{GnRH}$ ? Comment migrent-elles dans le cerveau? Quelle est leur distribution dans le cerveau? Et enfin, au nombre de combien sont-elles?

Afin de répondre à ces questions, nous avons entrepris pour la première fois, dans une étude publiée en 2016 [3], une analyse détaillée du système à GnRH à partir d'une large cohorte d'embryons humains au $1^{\text {er }}$ trimestrel. Dans cette étude, nous avons combiné des techniques d'immunohistochimie conventionnelles avec une reconstruction d'images en 3 dimensions (3D), à partir de coupes histologiques ou à partir de tissus embryonnaires intacts rendus transparents grâce à une technologie innovante. Nous avons montré de manière tout à fait surprenante que le nombre de neurones à $\mathrm{GnRH}$ présents dans le cerveau était bien plus élevé qu'attendu et que leur migration était disparate: seuls $20 \%$ de ces neurones colonisent I'hypothalamus, tandis que les $80 \%$ restants atteignent d'autres régions cérébrales, laissant supposer que la $\mathrm{GnRH}$ puisse avoir d'autres fonctions.

\footnotetext{
${ }^{1}$ Tous les embryons humains analysés ont été obtenus dans le cadre d'interruptions volontaires de grossesse pratiquées dans le service de gynécologie de l'hôpital Jeanne de Flandre, Lille, France, après obtention du consentement éclairé et écrit des patientes. Les tissus ont été mis à disposition conformément aux règles de bonne pratique relatives à la la conservation, la transformation et au transport de tissus humains (publiées le 29 décembre 1998). L'autorisation d'utiliser des tissus fœtaux humains non pathologiques a été obtenue auprès de l'Agence de la Biomédecine (protocole $n^{\circ}$ PFS16-002).
}

${ }^{1}$ Unité Inserm U1172, laboratoire du développement et plasticité du cerveau neuroendocrine, centre de recherche Jean-Pierre Aubert, 1, place de Verdun, 59045 Lille, France.

${ }^{2}$ Institut de biologie de la reproductionspermiologie-CECOS, CHU de Lille, avenue Eugène Avinée, 59037 Lille, France.

${ }^{3}$ Faculté de médecine, Université de Lille, FHU 1000 Days for Health, 59045 Lille, France. anne-laure.barbotin@chru-lille.fr paolo.giacobini@inserm.fr

\section{Ontogenèse des neurones à GnRH} et voies de migration

La plupart des vertébrés possèdent deux systèmes olfactifs anatomiquement distincts: le système olfactif principal, qui permet la détection d'odorants volatils, et un système olfactif accessoire, appelé système voméronasal (VNO), impliqué dans la détection de phéromones et dans la médiation des comportements sociaux et reproducteurs. Chez l'homme, les placodes olfactives se développent à partir d'un épaississement de l'ectoderme vers la $5^{e}$ semaine de développement embryonnaire. Les neurones à $\mathrm{GnRH}$ naissent au niveau de ces placodes, puis migrent vers le cerveau antérieur ${ }^{2}$ (dont une partie deviendra l'hypothalamus) en suivant le trajet des nerfs olfactifs, voméro-nasaux (VNN) et du nerf terminal (TN).

Nous avons, dans un premier temps, étudié les étapes de la morphogenèse conduisant à la formation du système olfactif/voméro-nasal et à l'apparition des premiers neurones à $\mathrm{GnRH}$. Nos résultats révèlent que les cellules du VNO (correspondant à une invagination médiane de la placode olfactive) pourraient être les précurseurs des neurones à $\mathrm{GnRH}$.

La migration des neurones à $\mathrm{GnRH}$ a été étudiée grâce à un nouveau procédé baptisé «3DISCO » (3-dimensional imaging of solvent cleared organs) [4]. Jusqu'à présent, notre connaissance du trajet des neurones à GnRH s'appuyait essentiellement sur l'analyse de coupes histologiques sériées à différents stades du développement embryonnaire, les techniques

\footnotetext{
${ }^{2}$ Le cerveau antérieur, ou prosencéphale, correspond au cours de l'embryogenèse à la vésicule antérieure qui va donner naissance au télencéphale (comprenant notamment le cortex) et au diencéphale (comprenant notamment le thalamus et l'hypothalamus).
} 

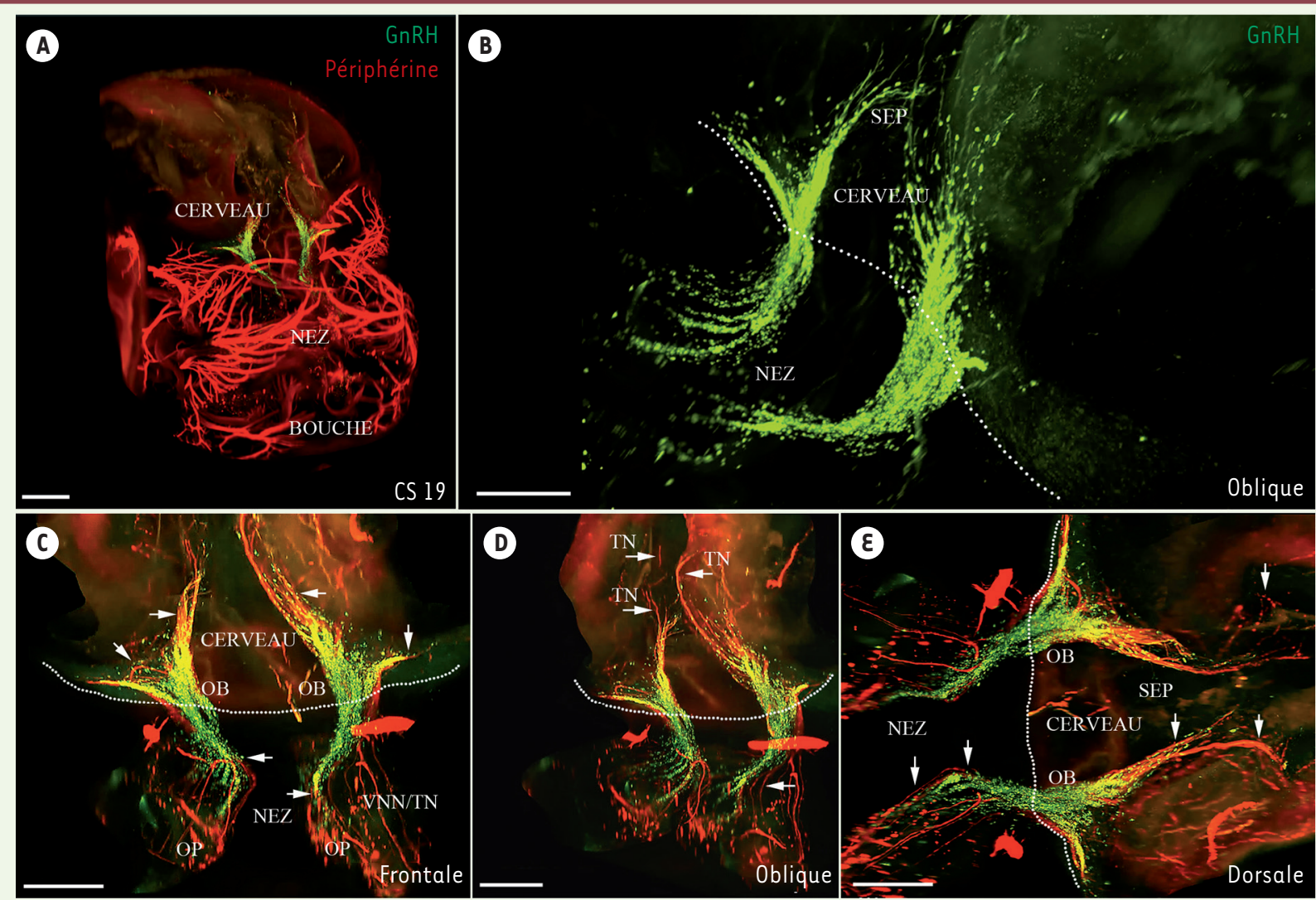

Figure 1. Visualisation en 3D d'un embryon humain préalablement rendu transparent au stade 19 de Carnegie (environ $48^{e}$ jour du développement embryonnaire). Les différentes vues (A-E) représentent la migration des neurones à GnRH (hormone de libération des gonadotrophines hypophysaires ; immunomarquage en vert) et la matrice de migration (immunomarquage par la périphérine en rouge). OP : placode olfactive ; VNN : nerf voméro-nasal; TN : nerf terminal ; OB : bulbe olfactif; SEP : aires septales. Les flèches indiquent les projections du VNN/TN. La ligne de pointillés indique la limite entre le nez et le cerveau. Échelles : A, $800 \mu \mathrm{m} ; \mathrm{B}, 200 \mu \mathrm{m} ; \mathrm{C}-\varepsilon, 300 \mu \mathrm{m}$ (๔) publié avec autorisation de Development (Cambridge, England) [3]).

d'imagerie optique usuelles ne permettant pas d'obtenir d'images à partir d'organes entiers en raison de l'opacité que leur confère leur épaisseur. Récemment, la technique 3DISCO a permis l'acquisition d'images à haute résolution à partir de tissus intacts (sans nécessité de coupes préalables des échantillons) après qu'ils aient été rendus transparents grâce à l'utilisation de solvants organiques [4]. Combinée à la réalisation d'un immunomarquage et à l'utilisation d'un ultramicroscope à feuille de lumière ${ }^{3}[5](\rightarrow)$,

\section{$(\rightarrow)$ Voir la Synthèse} de P.P. Girard et B.C. Forget, $m / s n^{\circ} 8-9$, août-septembre 2011, page 753

\footnotetext{
${ }^{3}$ Système permettant l'illumination et l'observation de
} tranches fines d'un échantillon. cette méthode permet d'étudier un fœtus dans son intégralité et donc en particulier le cerveau et la connectivité neuronale [6].

Grâce à l'application de cette nouvelle technologie couplée à un immunomarquage de la $\mathrm{GnRH}$ et de la périphérine ${ }^{4}$, qui marque l'ensemble des fibres du système nerveux périphérique, y compris celles qui sont utilisées par les neurones à GnRH comme support de migration (le nerf voméronasal et le nerf terminal), nous avons pu visualiser les neurones à $\mathrm{GnRH}$ en cours de migration dans la tête d'embryons humains au $19^{\mathrm{e}}$ stade de

${ }^{4}$ Protéine de la famille des filaments intermédiaires, spécifique du système nerveux périphérique.
Carnegie $^{5}$ (CS), équivalent au $48^{\mathrm{e}}$ jour de développement (Figure 1).

À ce stade de l'embryogenèse, un continuum bilatéral de neurones à $\mathrm{GnRH}$ en migration est visualisé entre la région nasale et le cerveau antérieur, se dirigeant vers le néocortex (NCX) et les aires septales $^{6}$ (SEP) (Figure IB). Nous avons observé, de façon inattendue, qu'en plus d'avoir déjà atteint les SEP, un sousensemble considérable de fibres du nerf terminal avait germé dorsalement et

\footnotetext{
${ }^{5}$ Les 8 semaines de la période embryonnaire ont été découpées en 23 stades, appelés «stades de Carnegie», sur la base de l'âge, la taille et les caractéristiques morphologiques de l'embryon.

${ }^{6}$ Région antérieure et médiale du lobe frontal, contenant plusieurs noyaux du système limbique (gérant les comportements émotionnels, mnésiques, la motivation, etc.).
} 


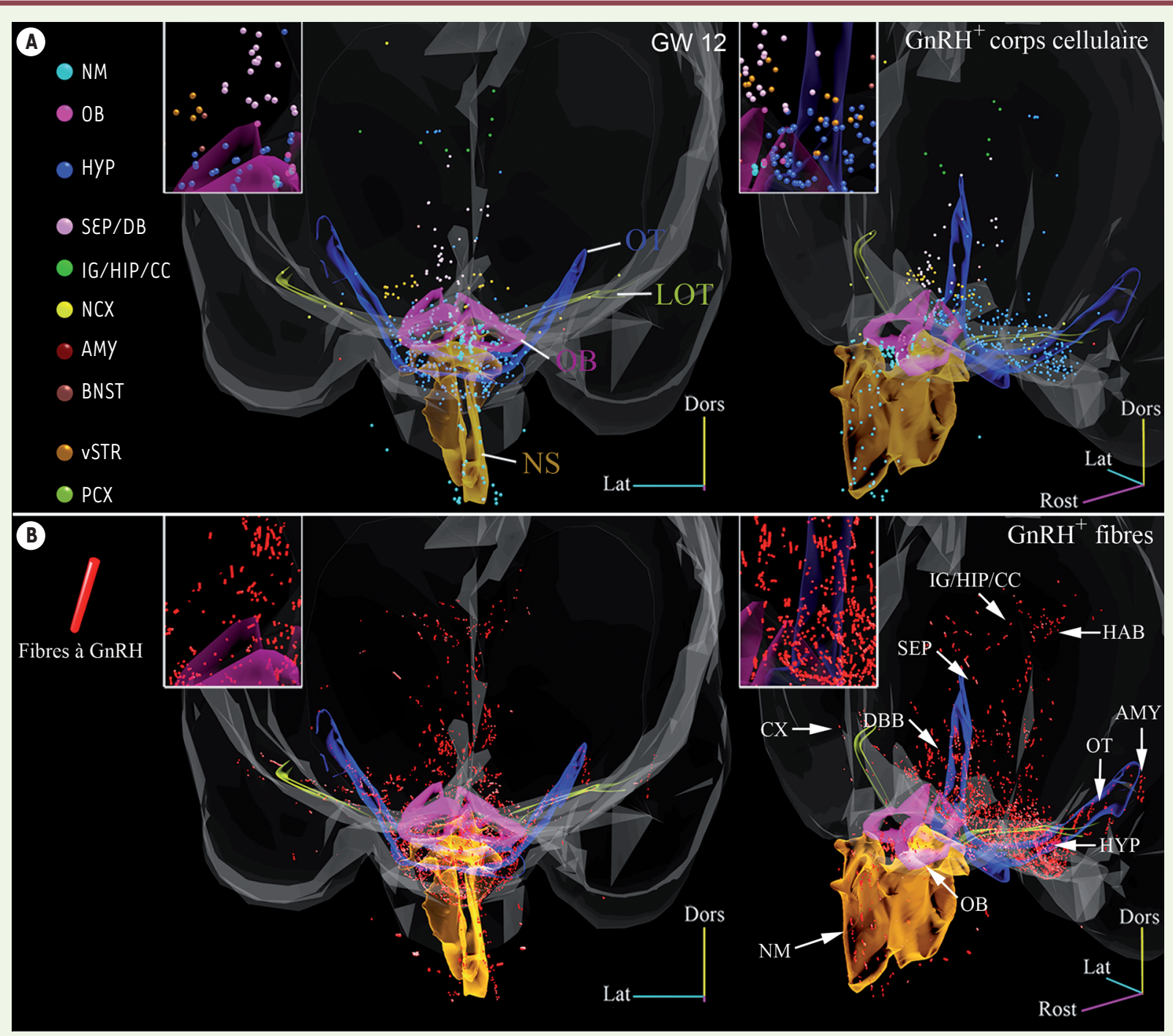

Figure 2. Distribution des corps cellulaires et des axones des neurones à GnRH chez un embryon humain à la 12e semaine. A. Vue avant (gauche) et perspective (droite) d'un modèle en 3D de distribution de corps cellulaires des neurones à GnRH (hormone de libération des gonadotrophines hypophysaires). Chaque cellule est représentée par une sphère (100 $\mu \mathrm{m}$ de diamètre) dont la couleur représente son emplacement (voir la légende à gauche). B. Même vue des modèles 3D que $A$ montrant la distribution des axones des neurones à GnRH (bâtonnets rouges). Les contours transparents du septum nasal (NS), du bulbe olfactif (OB), du tractus olfactif latéral (LOT) et du tractus optique (OT) sont également présentés à titre de référence. En encadré pour chaque vue : grossissement d'un petit détail du modèle 3D. NM : mésenchyme nasal ; HYP : hypothalamus ; SEP/DB : aires septales/bande diagonale de Broca ; IG/HIP/CC : indiseum griseum/hippocampe/circonvolution cingulaire; NCX : néocortex ; AMY : amygdale ; BNST : noyau des stria terminalis ; vSTR : striatum ventral ; PCX : cortex piriforme (๔ publié avec autorisation de Development (Cambridge, England) [3]).

latéralement vers des régions télencéphaliques ${ }^{2}$ correspondant au NCX et à I'hippocampe ${ }^{7}$ en développement. Alors que, chez les rongeurs, les neurones à $\mathrm{GnRH}$ migrant vers le cerveau antérieur semblent se détacher de leurs guides 7 Structure cérébrale appartenant au système limbique,
impliquée dans la mémoire. axonaux (constitués également par les fibres du nerf voméronasal et du nerf terminal) avant d'entrer dans la zone septo-préoptique [7], nous avons découvert que, chez l'humain, les neurones à $\mathrm{GnRH}$ restaient en association étroite avec ces fibres, tout au long de leur migration jusqu'à leur arrivée dans la région télencéphalique (Figure $1 C-\varepsilon$ ).
Cette observation suggère une différence fondamentale entre les rongeurs et les humains dans le mode de migration des neurones à GnRH. Par ailleurs, nous avons mis en évidence de manière inédite que les neurones à $\mathrm{GnRH}$ se répartissaient le long de deux flux migratoires distincts. En effet, en plus de la voie de migration ventrale se dirigeant vers les 
régions hypothalamiques [8], les neurones à $\mathrm{GnRH}$ suivent également une voie de migration dorsale orientée vers les régions télencéphaliques «palliale» et «sub-palliale ${ }^{8} »$. Cette nouvelle voie comprend deux composantes : une composante médiane et une autre composante latérale. Nous avons ainsi observé pour la première fois que des neurones à GnRH migraient en dehors de l'hypothalamus dans notre espèce.

\section{Quantification des neurones à GnRH}

Nous avons ensuite quantifié les cellules à $\mathrm{GnRH}$, à différents stades de l'embryogenèse entre le $39^{\mathrm{e}}$ jour (CS16) et la $12^{\mathrm{e}}$ semaine de développement. Au stade CS16, nous avons observé la présence de 50 neurones à $\mathrm{GnRH}$, tous confinés à la région nasale. Quelques jours plus tard, le nombre de neurones à GnRH était passé à $7951 \pm 2000$, indiquant que la différenciation neuronale complète a lieu entre le $39^{\mathrm{e}}$ et le $44^{\mathrm{e}}$ jour de développement. En moyenne, 10000 neurones étaient détectables dans le cerveau entre la $6^{\mathrm{e}}$ et la $12^{\mathrm{e}}$ semaine de l'embryogenèse alors que les précédents comptages estimaient leur nombre à $2000[9,10]$, ceux-ci ayant été réalisés sur des hypothalamus adultes.

\section{Distribution des neurones à GnRH}

Nous avons évalué la distribution relative des corps cellulaires des neurones à $\mathrm{GnRH}$, visualisés par 3DISCO, dans trois zones (Figure 2): la région nasale (leur région d'origine), la voie de migration ventrale et la voie dorsale. Au cours du développement embryonnaire, le nombre de cellules à $\mathrm{GnRH}$ présentes dans la région nasale diminue progressivement. Cette diminution est concomitante à une augmentation de leur nombre dans le cerveau au sein des composantes migratoires dorsales et ventrales. Ainsi, au dernier stade étudié ( $12^{\mathrm{e}}$ semaine),

\footnotetext{
${ }^{8}$ Le pallium correspond, au moment de l'embryogenèse, à ce qui va devenir le cortex cérébral. Les régions palliales sont donc les futures régions corticales, et les régions télencéphaliques sub-palliales, les futures régions sous-corticales du télencéphale.
}

seuls $23 \%$ des neurones à $\mathrm{GnRH}$ étaient retrouvés dans les régions les plus rostrales (région nasale/bulbe olfactif). Cette observation confirme que la migration des neurones à GnRH intervient assez précocement au cours l'embryogenèse (au cours du premier trimestre de gestation). Afin de mieux comprendre la localisation anatomique de ces cellules dans le cerveau à la $12^{\mathrm{e}}$ semaine de développement, nous avons procédé à un immunomarquage classique de la $\mathrm{GnRH}$ sur des coupes sériées de tissus et effectué une reconstruction en 3D (Figure 2). Cette expérience a confirmé la distribution étonnamment étendue des neurones et des fibres à GnRH dans l'hypothalamus, mais surtout, dans les régions extrahypothalamiques où est distribuée $80 \%$ de la population des neurones à $\mathrm{GnRH}$. Ces neurones sont en effet retrouvés, entre autres, dans le bulbe olfactif, le cortex cérébral, l'hippocampe, le cortex piriforme ${ }^{9}$, l'amygdale $^{10}$ et l'habenula ${ }^{11}$ (Figure $2 \mathrm{~A}$ et $2 \mathrm{~B}$ ).

\section{Conclusion}

Ce travail a mis en évidence que le nombre de neurones à $\mathrm{GnRH}$ était 5 fois plus élevé que ce qui était attendu et que l'hypothalamus fœtal n'était colonisé que par une petite fraction (20\%) de cette population de neurones. À la fin du $1^{\text {er }}$ trimestre, la grande majorité des neurones à GnRH (80\%) est distribuée dans des régions cérébrales extrahypothalamiques. Cet éclairage inédit sur notre connaissance de l'ontogenèse du système à $\mathrm{GnRH}$ a été rendu possible grâce à l'utilisation de nouvelles techniques d'imagerie. L'utilisation de la reconstruction en $3 D$ a notamment été mise à profit récemment afin d'élaborer le premier atlas en 3D couvrant les deux premiers mois du développement embryonnaire [11, 12]. Dans notre étude, cette approche a permis la réalisation d'une cartographie complète des corps

\footnotetext{
${ }^{9}$ Une des régions olfactives.

${ }^{10}$ Noyau cérébral impliqué dans le traitement des émotions.

${ }^{11}$ Structure appartenant à l'épithalamus.
}

cellulaires et des axones des neurones à GnRH à partir d'embryons humains pour la toute première fois. Bien que le devenir à long terme des neurones à $\mathrm{GnRH}$ extra-hypothalamiques reste inconnu, leur présence dans ces régions suggère qu'ils pourraient être impliqués dans des processus physiologiques autres que celui du contrôle de la reproduction, comme la cognition, les comportements ou encore le contrôle des émotions et les maladies associées. $\diamond$

Development of $\mathrm{GnRH}$ neurons in humans: new insights from 3D-imaging of fetal brains

\section{LIENS D'INTÉRÊT}

Les auteurs déclarent n'avoir aucun lien d'intérêt concernant les données publiées dans cet article.

\section{RÉFÉRENCES}

1. Christian CA, Moenter SM. The neurobiology of preovulatory and estradiol-induced gonadotropinreleasing hormone surges. Endocr Rev 2010 ; 31 : 544-77.

2. Catteau-Jonard S, Dewailly D, Prévot V, et al. L'hormone anti-müllerienne : une hormone ovarienne exerçant une rétroaction hypothalamique? Med Sci (Paris) $2016 ; 32: 441-4$.

3. Casoni F, Malone SA, Belle M, et al. Development of the neurons controlling fertility in humans: new insights from 3D imaging and transparent fetal brains. Development 2016 ; 143 : 3969-81.

4. Ertürk A, Becker K, Jährling N, et al. Three-dimensional imaging of solvent-cleared organs using 3DISCO. Nat Protoc $2012 ; 7$ : 1983-95.

5. Girard PP, Forget BP. Microscopie de fluorescence à feuille de lumière : La face cachée de l'échantillon enfin dévoilée. Med Sci (Paris) 2011 ; 27 : 753-62.

6. Belle M, Godefroy D, Dominici C, et al. A simple method for 3D analysis of immunolabeled axonal tracts in a transparent nervous system. Cell Rep 2014 ; 9: 1191-201.

7. Yoshida K, Tobet SA, Crandall JE, et al. The migration of luteinizing hormone-releasing hormone neurons in the developing rat is associated with a transient, caudal projection of the vomeronasal nerve.) Neurosci $1995 ; 15: 7769-77$.

8. Schwanzel-Fukuda M, Crossin KL, Pfaff DW, et al. Migration of luteinizing hormone-releasing hormone (LHRH) neurons in early human embryos. J Comp Neurol 1996 ; 366 : 547-57.

9. Crowley WF, Pitteloud N, Seminara S. New genes controlling human reproduction and how you find them. Trans Am Clin Climatol Assoc 2008 ; 119 : 29-37.

10. Tobet SA, Bless EP, Schwarting GA. Developmental aspect of the gonadotropin-releasing hormone system. Mol Cell Endocrinol 2001 ; 185 : 173-84.

11. de Bakker BS, de Jong KH, Hagoort J, et al. An interactive three-dimensional digital atlas and quantitative database of human development. Science 2016 ; 354. doi: 10.1126/science.aag0053.

12. Belle M, Godefroy D, Couly G, et al. Tridimensional visualization and analysis of early human development. Cell 2017 ; 169 : 161-73.e12. 